\title{
Rational design of 0D Mn0.05Cd0.95S anchored on 3D NiSe2 nanoparticles for efficient photocatalytic hydrogen production
}

\author{
Hua Liu ${ }^{1}$, Teng Yan ${ }^{1}$, Qingxiang $\mathrm{Ma}^{2}$, and Zhiliang $\mathrm{Jin}^{3}$ \\ ${ }^{1}$ North Minzu University \\ ${ }^{2}$ Ningxia University \\ ${ }^{3}$ Affiliation not available
}

June 8,2020

\begin{abstract}
A novel effective 0D/3D nanohybrids of Mn0.05Cd0.95S/NiSe2 photocatalysts, which are formed by Mn0.05Cd0.95S nanoparticles anchored on the outer surface of cubic NiSe2 in designed and prepared. The optimum hydrogen evolution rate of the Mn0.05Cd0.95S/NiSe2 composite is $14.3 \mathrm{mmol} \mathrm{h-1} \mathrm{g}-1$, which is 7.5 times higher than that of single Mn0.05Cd0.95S. Structural analyses and experimental results reveal that a little amount of NiSe2 can remarkably improve the photocatalytic activity and the close contact of the 0D/3D composite catalyst also plays an important role. Furthermore, NiSe2 can not only as both an electron acceptor to capture electrons and inhibit the recombination of electron-hole pairs, but also provide sufficient active sites to facilitate the photocatalytic water-splitting reaction activity. This work demonstrates that low-cost NiSe2 is a promising co-catalyst for photocatalytic splitting of water to produce hydrogen.
\end{abstract}

\section{Introduction}

In recent decades, the excessive use of fossil fuels has led to a battery of energy shortages and environmental pollution questions $[1,2]$. Compared with traditional fossil fuels, hydrogen is a clean and efficient energy carrier, since the $\mathrm{H}_{2}$ evolution from water splitting is one of the more promising methods with great promise to solve the problem $[3,4]$. Noble metal Pt not only has excellent electron absorption ability, but also provides abundant active sites, thus Pt has superior performance in terms of hydrogen production [5]. While high cost and preciousness of Pt limit its large-scale application in industry [6]. Hence, it is a challenging task to develop novel photocatalyst with excellent activity and low-cost to replace noble metals.

The various semiconductor photocatalysts have been extensively studied, such as metallic sulfide $\left(\mathrm{MoS}_{2}\right.$ [7], $\mathrm{CdS}$ [8], $\mathrm{ZnS}$ [9], $\mathrm{Cd}_{\mathrm{x}} \mathrm{Zn}_{1-\mathrm{x}} \mathrm{S}$ [10, 11]), metallic oxide $\left(\mathrm{Co}_{3} \mathrm{O}_{4}\right.$ [12], $\mathrm{Cu}_{2} \mathrm{O}$ [13]), carbon-based materials $\left(\mathrm{g}-\mathrm{C}_{3} \mathrm{~N}_{4}[14]\right)$ and so on over the past decade. Among them, $\mathrm{Mn}_{\mathrm{x}} \mathrm{Cd}_{1-\mathrm{x}} \mathrm{S}$ solid was first reported in 2010 [15], it has attracted widespread attention due to their strong light absorption and adjustable energy band structures $[16,17]$. Peculiarly, it overcomes the disadvantages of poor corrosion resistance and low activity of CdS, and it has highly efficient $\mathrm{H}_{2}$ production activity. However, pure $\mathrm{Mn}_{\mathrm{x}} \mathrm{Cd}_{1-\mathrm{x}} \mathrm{S}$ still exhibits severe recombination of photo-induced carriers that limited its activity in photocatalytic reaction [18]. Thus, many effective strategies have been presented to solve this problem, such as elemental doping [19], constructing heterojunction [20], morphological tailoring [21] and so on. Thereinto, co-catalyst not only provide enough redox reaction sites, but also reduce activation potentials and accelerate the transfer of photo-generated electrons [22]. For example, Liu's et al. [23] utilised the CoP nanoparticles to modify $\mathrm{Mn}_{0.2} \mathrm{Cd}_{0.8} \mathrm{~S}$ nanowires and significantly improved hydrogen production activity. 
The mainly Ni-based co-catalysts were widely developed in the field of photocatalytic hydrogen production $[24,25]$, including $\mathrm{Ni}_{2} \mathrm{P}, \mathrm{NiS}_{\mathrm{x}}$ and $\mathrm{Ni}(\mathrm{OH})_{2}$ etc. As one of the Ni-based materials, $\mathrm{NiSe}_{2}$ collects the advantages of high light absorption conversion ability, reduced band gap and remarkable conductivity in terms of photoelectricity [26, 27]. Secondly, it has low cost and yields more [28, 29]. Chen and co-workers [30] used CdS and $\mathrm{NiSe}_{2}$ to build a p-n heterojunction to improve hydrogen production activity. However, $\mathrm{NiSe}_{2}$ has been widely studied mostly in the field of eletrocatalysis, and there are few reports on photocatalytic hydrogen production. In addition to the strategy of adding co-catalysts, by adjusting and controlling the morphology, size and microstructure of nanomaterials, the composite material giving rising to distinct optical and mechanical properties, which is also a promising optimization method [31]. The hierarchical three dimensional (3D) structures could availably inhibit the stacking or aggregation of nanoparticles than two dimensional (2D) nanosheet materials [32]. On the other hand, the surface of the 3D structure can provide a large number of reaction sites and promote charge transfer efficiency [31], which enhanced to the photocatalytic activity.

Inspired by aforementioned discussion, in this work, we developed a facile method to incorporate 0D $\mathrm{Mn}_{0.05} \mathrm{Cd}_{0.95} \mathrm{~S}$ into 3D $\mathrm{NiSe}_{2}$. Through a series of detailed characterizations of the composition, microstructure, BET surface areas and optical properties of $\mathrm{Mn}_{0.05} \mathrm{Cd}_{0.95} \mathrm{~S} / \mathrm{NiSe}_{2}$ composites, the effect of $\mathrm{NiSe}_{2}$ was researched on the activity of material. In addition, we proposed a possible mechanism of $\mathrm{Mn}_{0.05} \mathrm{Cd}_{0.95} \mathrm{~S} / \mathrm{NiSe}_{2}$ composite based on the conclusions of photoelectrochemical measurements and photoluminescence spectroscopy.

\section{Experimental Section}

\subsection{Materials}

All chemicals used in this experiment were of analytical grade and used without further purification.

\subsubsection{Preparation of $\mathrm{Mn}_{0.05} \mathrm{Cd}_{0.95} \mathrm{~S}$}

The $\mathrm{Mn}_{0.05} \mathrm{Cd}_{0.95} \mathrm{~S}$ solid solutions were synthesized according to the previous report [33]. In a typical process, manganese acetate tetrahydrate $(0.44 \mathrm{~g})$, cadmium acetate anhydrous $(1.919 \mathrm{~g})$ and thioacetamide $(2.5 \mathrm{~g})$ were added into $20 \mathrm{~mL}$ of ethylenediamine and $40 \mathrm{~mL}$ deionized water and dispersed by sonication for 20 min. After stirring for $1.5 \mathrm{~h}$, the mixture solution was transferred into a Teflon-lined autoclave $(80 \mathrm{~mL})$, the autoclave was maintained at $160{ }^{\circ} \mathrm{C}$ for $24 \mathrm{~h}$. After cooling naturelly, the yellow sediment was centrifuged and washed by deionized water and absolute ethyl alcohol respectively and then dried at $60{ }^{\circ} \mathrm{C}$ for $10 \mathrm{~h}$ to get the $\mathrm{Mn}_{0.05} \mathrm{Cd}_{0.95} \mathrm{~S}$ composite which named as MCS.

\subsubsection{Preparation of $\mathrm{NiSe}_{2}$}

In a typical synthesis, the selenium powder $(1.56 \mathrm{~g})$ was first dissolved in $6 \mathrm{~mL}$ hydrazine hydrate and $54 \mathrm{~mL}$ deionized water and ultrasonic treatment for $30 \mathrm{~min}$. Then, nickel nitrate hexahydrate $(2.88 \mathrm{~g})$ was joined into the above solution and drastic stirred for $30 \mathrm{~min}$. The resultant suspension was heat-treated at $180^{\circ} \mathrm{C}$ for $24 \mathrm{~h}$. The subsequent processing steps are consistent with preparation of MCS.

\subsubsection{Preparation of $\mathrm{Mn}_{0.05} \mathrm{Cd}_{0.95} \mathrm{~S} / \mathrm{NiSe}_{2}$}

The MCS nanoparticles were anchored on the surface of $\mathrm{NiSe}_{2}$ by a simple physical mixing method. The MCS (2 g) was added into $30 \mathrm{~mL}$ ethanol and dispersed by sonication for $30 \mathrm{~min}$. Then add a certain amount of $\mathrm{NiSe}_{2}$ to the above solution and stirred for $30 \mathrm{~min}$. The mixed solution was evaporated by constant stirring at $80{ }^{\circ} \mathrm{C}$. The resulting samples were filtered and washed with water and alcohol. The ultimate $\mathrm{Mn}_{0.05} \mathrm{Cd}_{0.95} \mathrm{~S} / \mathrm{NiSe}_{2}$ products were labeled as MCNS-x (where $\mathrm{x}$ is displayed by the nominal weight percentage (wt \%) of $\mathrm{NiSe}_{2}$ in $\mathrm{Mn}_{0.05} \mathrm{Cd}_{0.95} \mathrm{~S}$ ).

\subsection{Characterization of samples}

The morphologies of the samples were measured by field emission scanning electron microscope (SEM: JSM6701F. JEOL) and transmission electron microscope (TEM: FEI Tecnai TF 20). The crystalline structure 
was analyzed by X-ray diffraction (XRD: Rigaku RINT-2000) patterns, all samples were scanned from $5^{\circ}$ to $80^{\circ}$ at a scanning rate of $5^{\circ} / \mathrm{min}$ under $40 \mathrm{~mA}$ and $40 \mathrm{kV}$. The chemical composition and valence state of the catalyst were detected by X-ray photoelectron (XPS: ESCALAB 250Xi). UV-vis diffuse reflectance spectra (DRS) were performed by Shimadzu UV 2550 spectrophotometer with $\mathrm{BaSO}_{4}$ powder was used as a reference. Photoluminescence (PL) spectra and time resolved photoluminescence (TRPL) spectra were analyzed on a FIUOROMAX-4 spectrophotometer (HORIBA Scientific, France) with the excitation wavelength of $390 \mathrm{~nm}$. Brunauer-Emmett-Teller (BET) surface area and $\mathrm{N}_{2}$ adsorption-desorption isotherms were carried out on an ASAP2020M instrument at $77 \mathrm{~K}$.

The electrochemical tests and photoelectrochemical properties of MCS, NiSe 2 and MCNS-10 samples were performed by an electrochemical workstation (VersaSTA4-400, AMETEK). The transparent solution of $\mathrm{Na}_{2} \mathrm{SO}_{4}(0.2 \mathrm{M})$ was used as electrolyte. In this test system, a platinum sheet was used as the counter electrode and reference electrode of saturated calomel electrode (SCE) and the work electrode of as-prepared was the fluorine-doped tin oxide (FTO) coated. The FTO working electrodes was prepared as follows: the FTO glass were cut to $2 \times 1 \mathrm{~cm}^{2}$, it was orderly washed with acetone, ethanol and deionized water in ultrasonic devices. $5 \mathrm{mg}$ catalyst was ultrasonically dispersion for $1 \mathrm{~h}$ in $100 \mu \mathrm{L}$ anhydrous ethanol containing 10 $\mu \mathrm{L}$ of $5 \%$ Nafion solution. Then the dispersed solutions of catalyst was uniformly coated FTO glass with the areas about $1 \mathrm{~cm}^{2}$ until it dried. The linear sweep voltammetry (LSV) and electrochemical impedance spectroscopy (EIS) were measured at room temperature with a scan rate of $0.05 \mathrm{~V} \mathrm{~s}^{-1}$. When the forward bias is $0.2 \mathrm{~V}$, the EIS of samples were measured in a frequency range of $1 \mathrm{MHz}$ to $1 \mathrm{~Hz}$ at $\mathrm{AC}$ amplitude of $10 \mathrm{mV}$. Mott-Schottky was tested under the frequency of $1000 \mathrm{~Hz}$.

\subsection{Photocatalytic evaluation}

In a $63 \mathrm{~mL}$ sealed quartz bottle, $0.01 \mathrm{~g}$ catalyst was dissolved in $30 \mathrm{~mL} \mathrm{Na} 2 \mathrm{~S} / \mathrm{Na}_{2} \mathrm{SO}_{3}(0.35 \mathrm{M} / 0.25 \mathrm{M}) . \mathrm{N}_{2}$ was used replace the oxygen in the bottle, the exhaust process takes about $5 \mathrm{~min}$. Then the treated quartz bottle was put into a nine-channel photocatalytic reaction system (PCX50A Discover) for hydrogen evolution experiment. Under the illumination of $5 \mathrm{~W}$ LED white light, extract $0.5 \mathrm{ml}$ of gas from the bottle every hour and injected it into the Tianmei GC7900 gas chromatograph (TCD, 13Xcolumn) with $\mathrm{N}_{2}$ as carrier gas, the hydrogen content in the gas is identified and recorded. Moreover, the apparent quantum efficiency (AQE) of the MCNS-10 was measured under a $300 \mathrm{~W}$ xenon lamp. The light source was transmitted through filters with specifications of 400,420,450,475, 500, 520, 550 and $600 \mathrm{~nm}$, respectively. The number of incident photons fluxes was tested using a power meter (PL-MW200 radiometer). The calculation formula for AQE was as follows at different wavelengths.

\section{Results and discussion}

\subsection{XRD analysis}

Fig. 1 depicts the crystal structure and crystallinity of pure MCS, $\mathrm{NiSe}_{2}$ and $\mathrm{MCNS}$ composites by XRD analysis. In Fig. 1a , seven distinct diffraction peaks of pure MCS at $24.92^{\circ}, 26.65^{\circ}, 28.37^{\circ}, 36.80^{\circ}$, $43.91^{\circ}, 48.08^{\circ}$ and $52.04^{\circ}$ correspond to the crystal planes of (100), (002), (101), (102), (110), (103) and (114) planes, respectively. The diffraction peaks of $\mathrm{NiSe}_{2}$ was corresponded to the cubic $\mathrm{NiSe}_{2}$ with the well matched (200), (210), (211), (311), (023) and (321) crystal planes, respectively. The strong and sharp characteristic peaks of $\mathrm{NiSe}_{2}$ suggested high crystallinity of $\mathrm{NiSe}_{2}$ synthesized by hydrothermal method. No other peaks originating by impurities were observed, in the XRD patterns of MCS and $\mathrm{NiSe}_{2}$ alone, implying the successful preparation of MCS solid solution and $\mathrm{NiSe}_{2}$. In theFig. 1 $\mathbf{b}$, the diffraction peaks of composite catalyst were actually resembled to pure MCS, indicating that adulteration with a little amount of $\mathrm{NiSe}_{2}$ had not effected on the crystal phase of MCS. Besides, the intensity of diffraction peaks of $\mathrm{NiSe}_{2}$ gradually strengthened with increased of $\mathrm{NiSe}_{2}$, indicating the successfully fabricated of composite catalyst. 

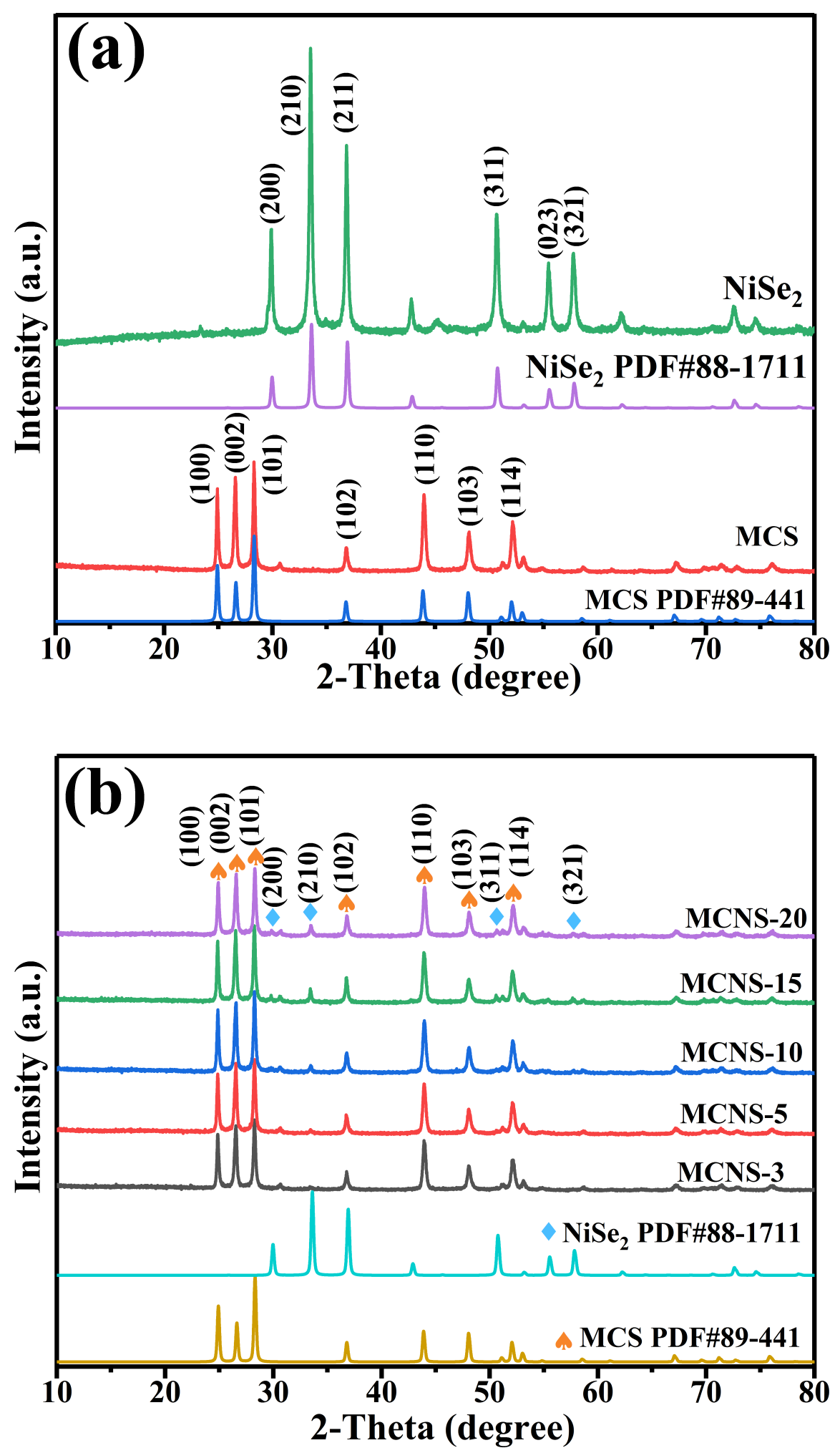

Fig. 1 XRD patterns of (a) MCS, $\mathrm{NiSe}_{2}$ and (b) MCNS composites with different weight loading ratios of $\mathrm{NiSe}_{2}$. 


\subsection{SEM and TEM analysis}

The morphology and microstructure of MCS, $\mathrm{NiSe}_{2}$ and MCNS-10 samples were investigated by SEM and high-resolution TEM. As shown in Fig. 2a , the prepared MCS solid solutions were irregular particles and agglomeration agglomeration, which was consistent with the morphologies reported in previous literature [33]. In Fig. 2b , the $\mathrm{NiSe}_{2}$ was obviously observed to be greater irregular crystals than that MCS, some of them were cubic crystal structures consistent with XRD pattern. The TEM image (Fig. 2c) showed some MCS nanoparticles were anchored on the surface of cubic $\mathrm{NiSe}_{2}$. Compared with pure MCS, the dispersion of MCS has been greatly improved in the composite catalyst, this intimate interface contact was conductive to separation and transfer of electron-hole pairs. HRTEM image in Fig. 2d shown the lattice fringes of the MCNS-10, the crystal plane spacing of $0.357 \mathrm{~nm}$ (red mark) and $0.298 \mathrm{~nm}$ (blue mark) were respectively corresponded to the (100) plane of hexagonal MCS and (200) plane of cubic $\mathrm{NiSe}_{2}$. Fig. 2e shown the EDX spectrum of the MCNS-10 which demonstrated the existence of Mn, Cd, S, Ni and Se elements, so the successful preparation of MCNS-10 may be proved. Further, the $\mathrm{Cu}$ element in the spectrum was mainly derived from the $\mathrm{Cu}$ carriers [34].


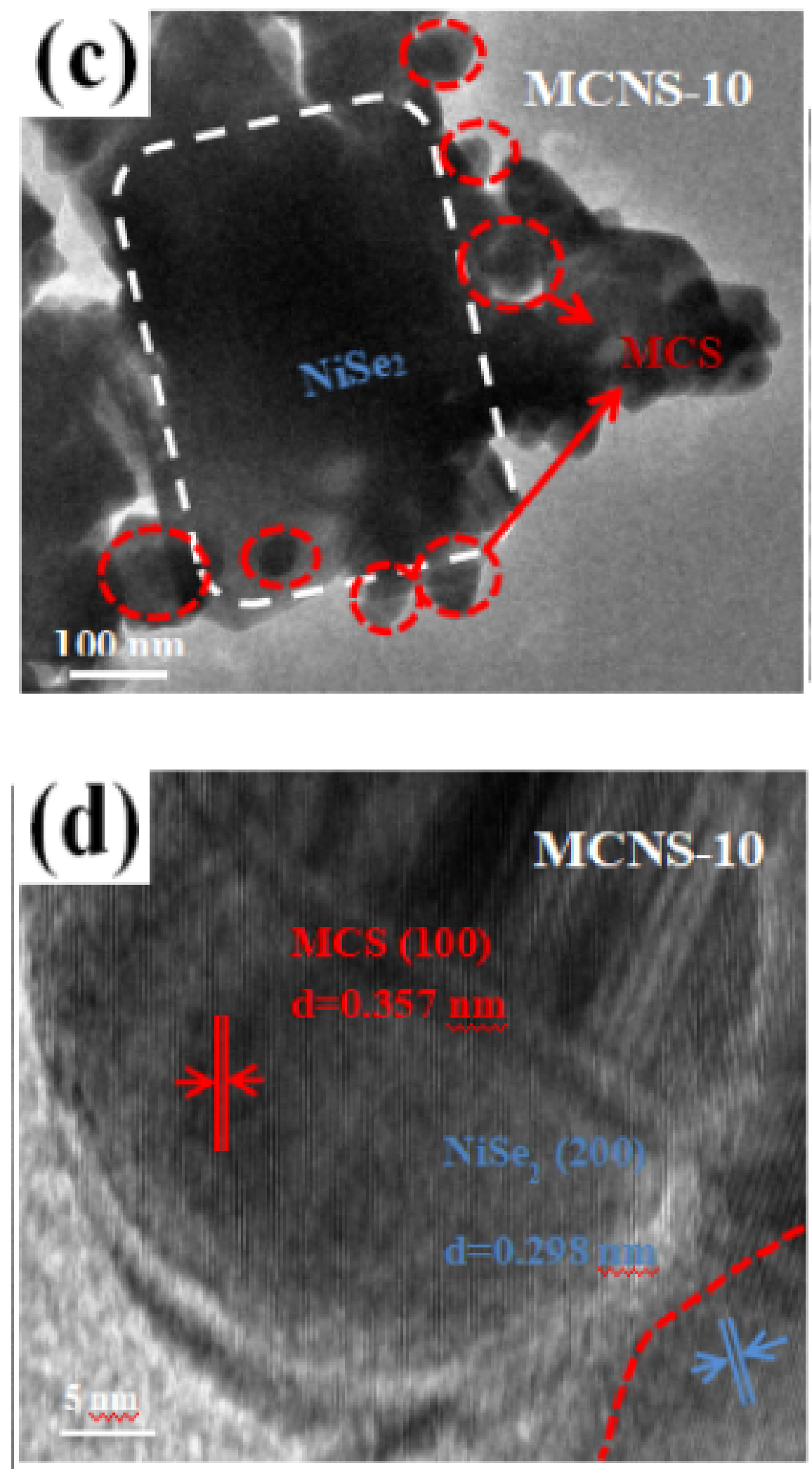


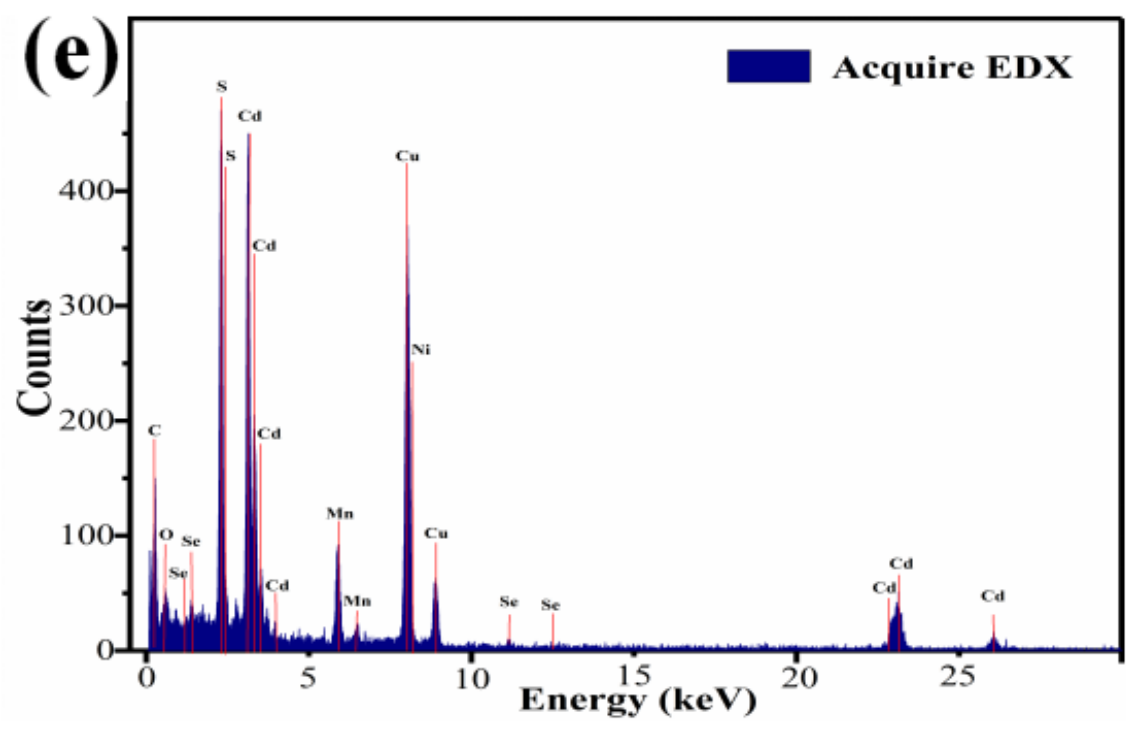

Fig. 2 (a) SEM images of pure MCS and (b)NiSe 2 ; (c) TEM images and (d) HR-TEM images of MCNS-10; (e) the EDX spectrum of MCNS-10.

\subsection{XPS analysis}

The X-ray photoelectron spectroscopy (XPS) was used to investigate the surface chemical states of MCNS10 composite. The Fig. 3aclearly reveals the existence of Mn, Cd, S, Ni and Se elements in MCNS-10 composite. Fig. 3b was the spectrum of $\mathrm{Cd} \mathrm{3d}$, the binding energies located at 404.6 and $411.3 \mathrm{eV}$ were respectively corresponded to $\mathrm{Cd} 3 \mathrm{~d}_{5 / 2}$ and $\mathrm{Cd} 3 \mathrm{~d}_{3 / 2}$, which were the $\mathrm{Cd}^{2+}$ ions [35]. The two binding energy of $\mathrm{Mn} 2 \mathrm{p}$ at 641 and $651.9 \mathrm{eV}$ belong to the $\mathrm{Mn} 2 \mathrm{p}_{3 / 2}$ and $\mathrm{Mn} 2 \mathrm{p}_{1 / 2}$ respectively as shown in Fig. 3c . According to the S 2p spectra shown in Fig. 3d , the peaks at $160.9\left(\mathrm{~S} \mathrm{2} \mathrm{p}_{3 / 2}\right)$ and $162.1\left(\mathrm{~S} 2 \mathrm{p}_{1 / 2}\right) \mathrm{eV}$ were the characteristic peaks of $\mathrm{S}^{2-}$ ions [33]. The deconvolution of the overlapped $\mathrm{Ni} 2 \mathrm{p}$ peaks four peaks (Fig. 3e ), the peaks located at 861.9 and $879.5 \mathrm{eV}$ were respectively related to the satellite peaks of the $\mathrm{Ni}$ $2 \mathrm{p}_{1 / 2}$ and $\mathrm{Ni} 2 \mathrm{p}_{3 / 2}$ [37], and the peaks centered at 855.4 and $873.2 \mathrm{eV}$ could be assigned to $\mathrm{Ni}^{2+}$ state [38]. The XPS spectrum of Se 3d represented in Fig. 3f could divided into three peaks, the main peaks located at 54.1 and $58.7 \mathrm{eV}$ were respectively related to $\mathrm{Se}^{2-}$ anions and high-valent oxide [39], and peak at $55 \mathrm{eV}$ were ascribed to Se $3 \mathrm{~d}_{5 / 2}$ of MCNS-10 [40]. These surface bonding states further proved that composite was composed of MCS and $\mathrm{NiSe}_{2}$. 

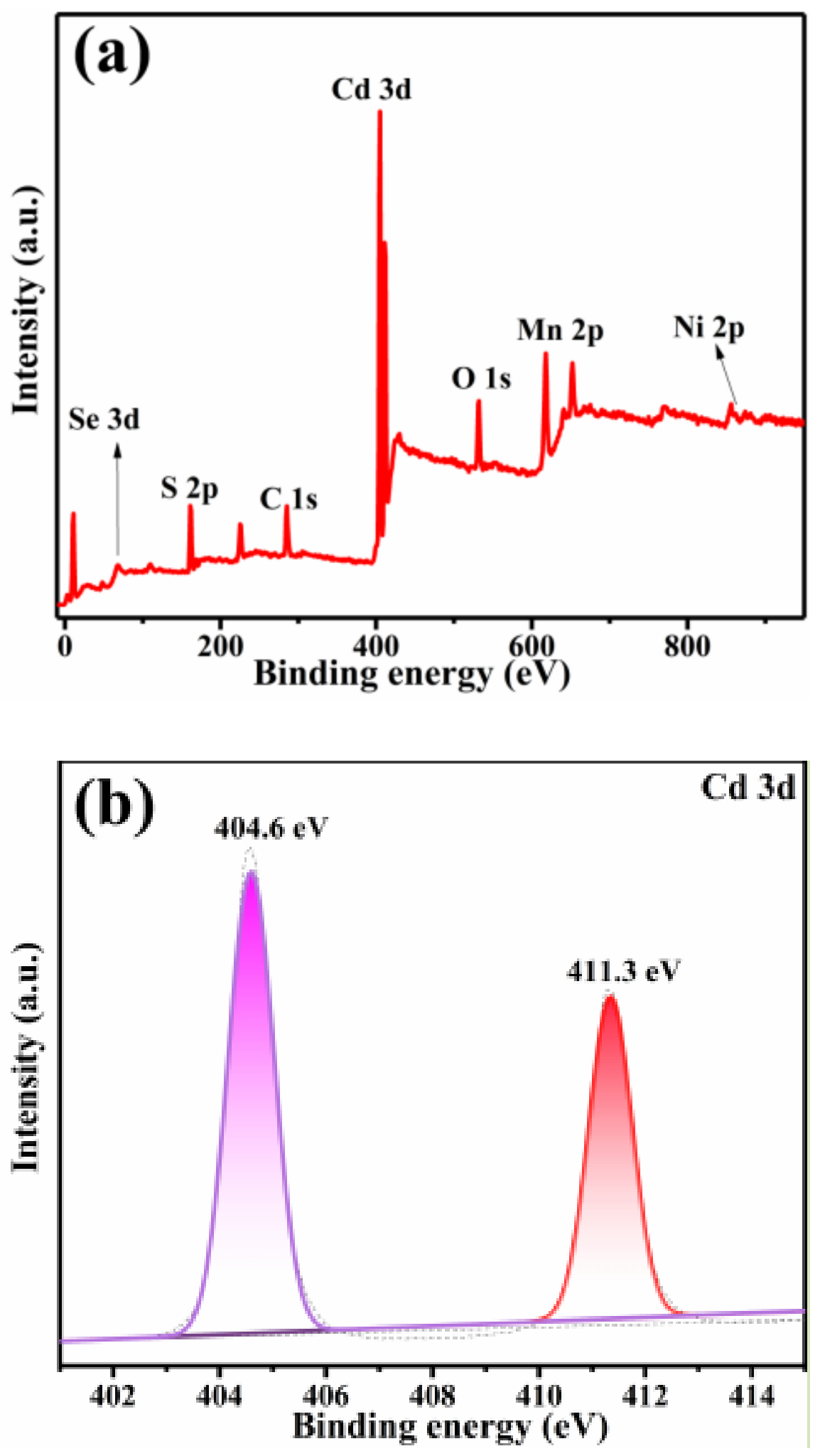

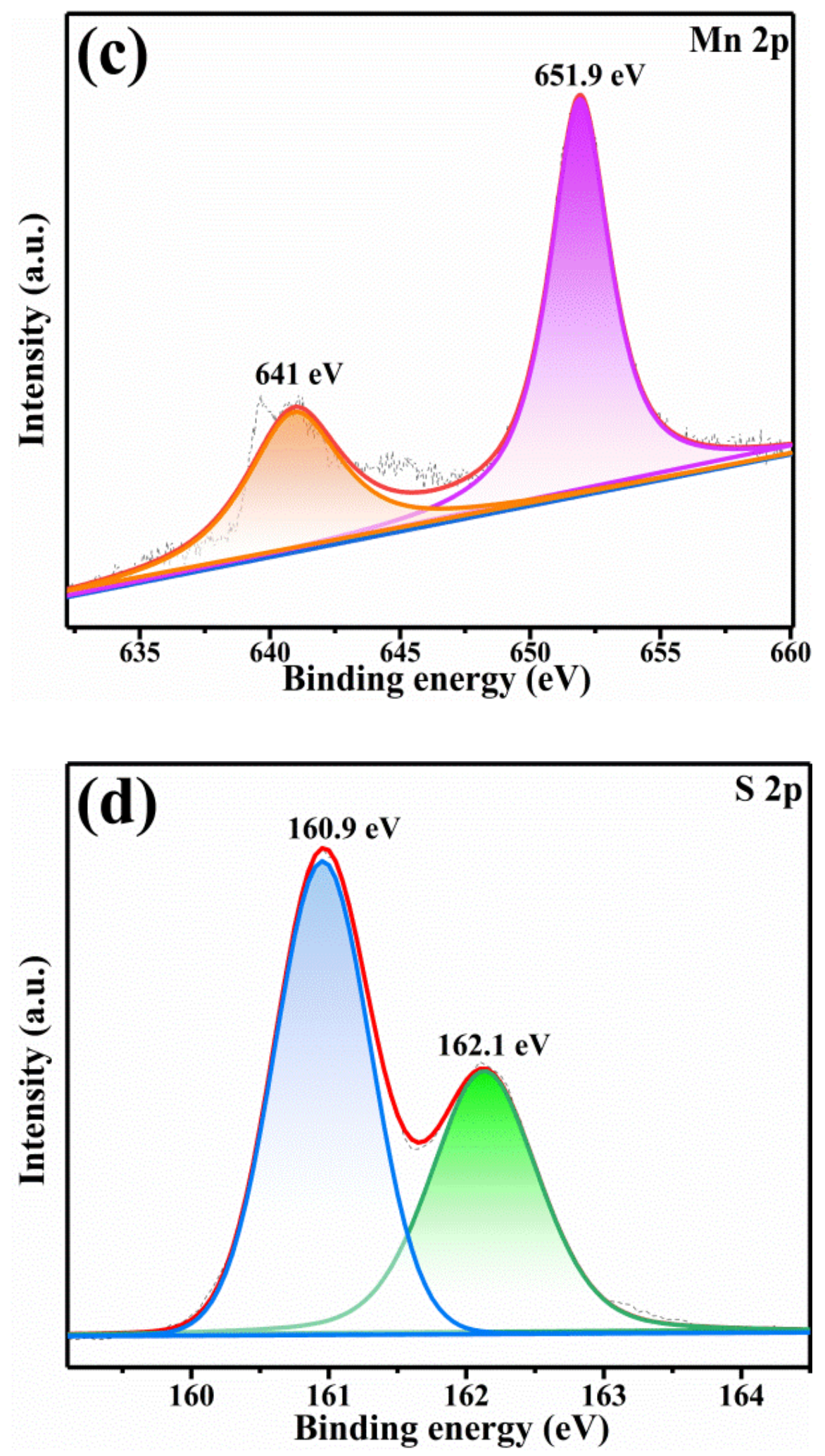



Fig. 3 XPS spectra of (a) survey, (b) Cd 3d,(c) Mn 2p, (d) S 2p, (e) Ni 2p and(f) Se 3d for MCNS-10 
composite.

\subsection{UV-vis DRS analysis}

A comparison of the UV-vis diffuse reflection spectroscopy (DRS) of MCS, $\mathrm{NiSe}_{2}$, MCNS composite were displayed in Fig. 4a . Pure $\mathrm{NiSe}_{2}$ exhibited high adsorption coefficient throughout in the visible light and near infrared regions, which was related to the metallic character [33]. The absorption edge of pure MCS solid solution was approximately $560 \mathrm{~nm}$. With the increasing of $\mathrm{NiSe}_{2}$ amount, the absorption intensity peak for composite catalyst was obviously increased at the 520-800 $\mathrm{nm}$ regions, which were ascribed to intrinsic absorption of $\mathrm{NiSe}_{2}$ and gradual deepening of the catalyst color. The light absorption efficiency and photocatalytic performance of composite catalyst were thus vastly improved [41], which was also one of the most important reasons for the improved hydrogen production performance of the catalyst. In addition, Fig. 4b present the corresponding energy band gaps $\left(\mathrm{E}_{\mathrm{g}}\right)$ of MCS and MCNS-10 were estimated by drawing on the Tauc plots of $(\mathrm{ahv})^{2}$ versus $h v$. The corresponding energy band gap values of MCS and MCNS-10 were estimated to be 2.26 and $2.2 \mathrm{eV}$, respectively. It could be seen that the improvement of the light absorption performance was related to the decrease of the band gap values.

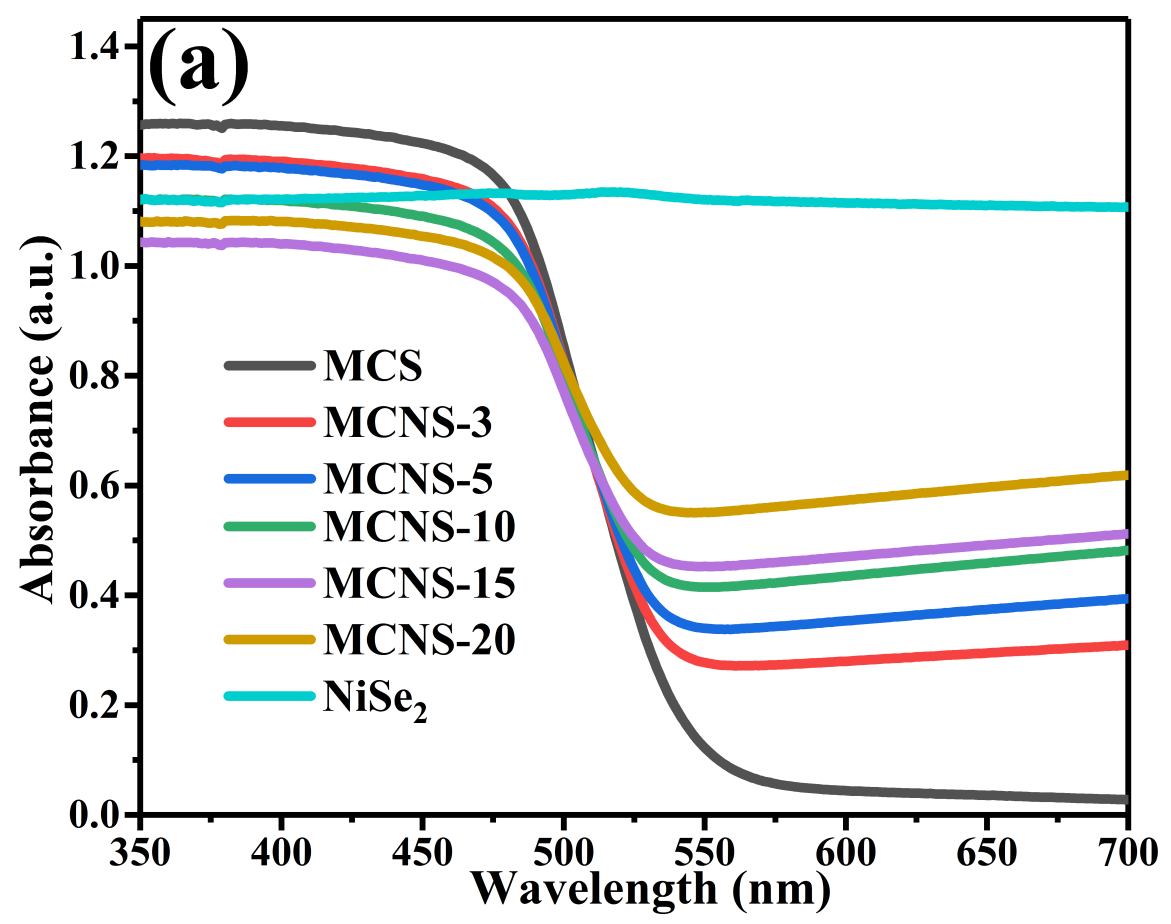






Fig. 4 (a) UV-vis DRS of MCS, NiSe 2 , MCNS-3, MCNS-5, MCNS-10, MCNS-15 and MCNS-20, (b) the forbidden band width of MCS, $\mathrm{NiSe}_{2}$ and MCNS-10.

\subsection{BET surface areas and pore size distributions}

The calculation results of Brunauer-Emmett-Teller (BET) specific surface area $\left(\mathrm{S}_{\mathrm{BET}}\right)$ and average pore size and pore volume of pure MCS, $\mathrm{NiSe}_{2}$ and MCNS-10 samples by $\mathrm{N}_{2}$ adsorption-desorption isotherms at 77 $\mathrm{K}$ as shownFig. $\mathbf{5}$ and Table $\mathbf{1}$. As shown inFig. $\mathbf{5}$, the all samples exhibited IV type isotherms with H3 hysteresis loops, indicating that they had mesoporous structure [42]. The $\mathrm{S}_{\mathrm{BET}}$ was augmented from 12 to $15 \mathrm{~m}^{2} \mathrm{~g}^{-1}$ after loading MCS onto surface of $\mathrm{NiSe}_{2}$, this was because the cubic $\mathrm{NiSe}_{2}$ provide enough space for anchoring of MCS nanoparticles, which effectively prevent the occurrence of agglomeration. In addition, the increased surface area of composite catalyst MCNS-10 exposing more activity sites, which enhancing photocatalytic hydrogen evolution performance [43]. In short, the change of $\mathrm{S}_{\mathrm{BET}}$ of MCNS-10 was consistent with the alter in morphology for MCNS-10 composite in TEM images. 




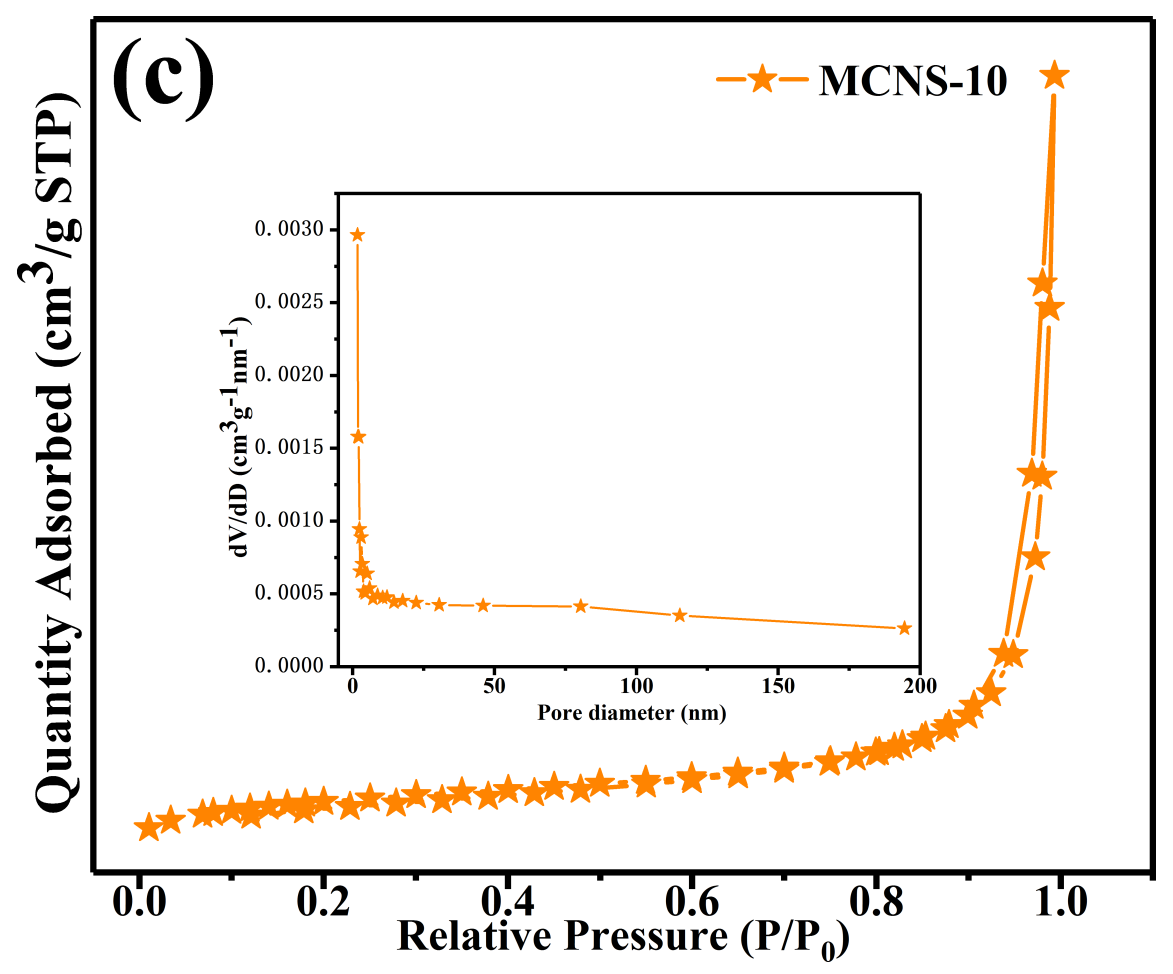

Fig. 5 The $\mathrm{N}_{2}$ absorption-desorption isotherms and corresponding pore size distribution of the (a) MCS, (b) $\mathrm{NiSe}_{2}$ and (c) MCNS-10.

Table 1 The physical adsorption performance parameters.

\begin{tabular}{llll}
\hline Samples & $\mathrm{S}_{\mathrm{BET}}\left(\mathrm{m}^{2} \mathrm{~g}^{-1}\right)^{\mathrm{a}}$ & Pore volume $\left(\mathrm{cm}^{3} \mathrm{~g}^{-1}\right)^{\mathrm{b}}$ & Average pore size $(\mathrm{nm})^{\mathrm{b}}$ \\
\hline MCS & 12 & 0.1 & 32 \\
$\mathrm{NiSe}_{2}$ & 8 & 0.03 & 13 \\
MCNS-10 & 15 & 0.01 & 28 \\
\hline
\end{tabular}

a Obtained from BET method;

b Relative pressure $\left(\mathrm{P} / \mathrm{P}_{0}\right)$ was 0.99 .

\subsection{Photocatalytic activity and stability}

Fig. 6 shown the photocatalytic $\mathrm{H}_{2}$ evolution performances of diverse catalysts with $\mathrm{Na}_{2} \mathrm{~S} / \mathrm{Na}_{2} \mathrm{SO}_{3}(0.35 \mathrm{M} / 0.25 \mathrm{M})$ solution as a sacrificial agent under visible light irradiation $(\lambda>400$ nm). InFig. 6a , the hydrogen production activity for bare $\mathrm{NiSe}_{2}$ was not detected, which manifested that it could not generate charge carries and had no photocatalytic activity. The MCS reveals poor photocatalytic activity $(95.74 \mu \mathrm{mol})$ when the irradiation time lasted for $5 \mathrm{~h}$. The $\mathrm{H}_{2}$ evolution rate of MCNS-x first increased and then decreased with the added amount of loaded $\mathrm{NiSe}_{2}$ from 3 to 20 wt\%. Obviously, the maximal hydrogen production amount obtained of $713.78 \mu \mathrm{mol}$ when the $\mathrm{NiSe}_{2}$ content was $10 \%$, increased by a factor of around 7.5 in comparison to pure MCS. However, the $\mathrm{H}_{2}$ production activity start to decrease when the content of $\mathrm{NiSe}_{2}$ exceed $10 \%$, which may be that the shielding effect of the mixed catalyst and the lack of hydrogen production activity of $\mathrm{NiSe}_{2}$ [33]. Besides, apparent quantum efficiency (AQE) and hydrogen evolution stability of MCNS-10 were important indicators to measure the practical application of catalysts.Fig. $\mathbf{6 b}$ shown the AQE value of MCNS-10 under different monochromatic light 
wavelengths. Th AQE and the intensity of UV absorption were basically the same, confirming that the activity of hydrogen production was related to the wavelength of incident light [44].

The cyclic tests of $\mathrm{H}_{2}$ production of MCS and MCNS-10 were performed in Fig. 6c . Compared with the composite catalyst MCNS-10, the total hydrogen production of MCS was basically unchanged, indicating that MCS has good stability. The hydrogen production activity of the composite catalyst MCS decreased significantly, the reason for this phenomenon may be increased in reaction time, which would exacerbate consumption of $\mathrm{Na}_{2} \mathrm{~S} / \mathrm{Na}_{2} \mathrm{SO}_{3}(0.35 \mathrm{M} / 0.25 \mathrm{M})$ solution [4]. Secondly, this was probably due to partial detachment of MCS and $\mathrm{NiSe}_{2}$ during the reaction. Fig. 6d compares the XRD patterns of composite catalyst MCNS-10 original and recycled hydrogen production. The position of diffraction peak was no significant change, but the intensities was reduced by half, which indicated that the crystallinity of the catalyst became worse.





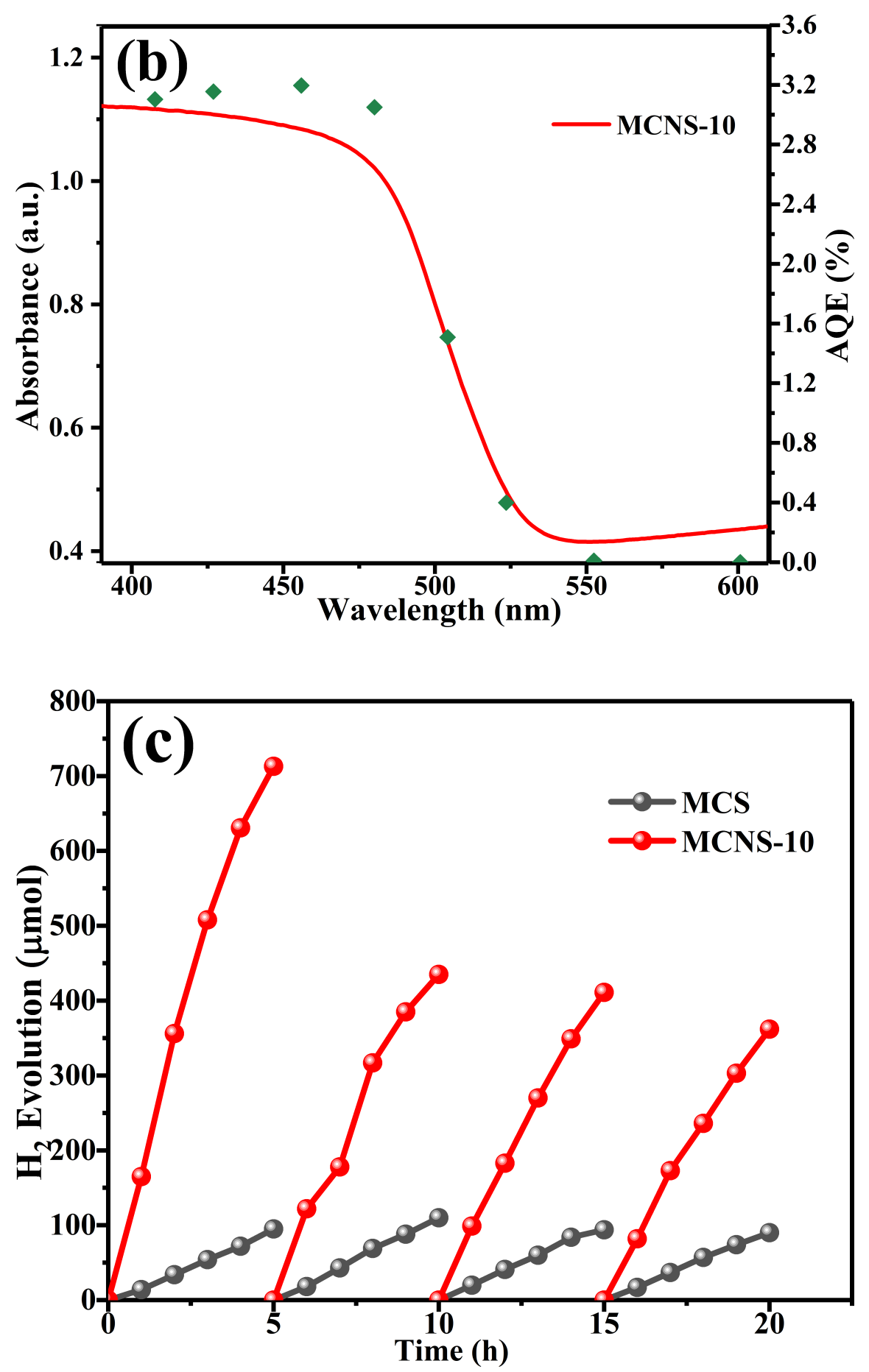


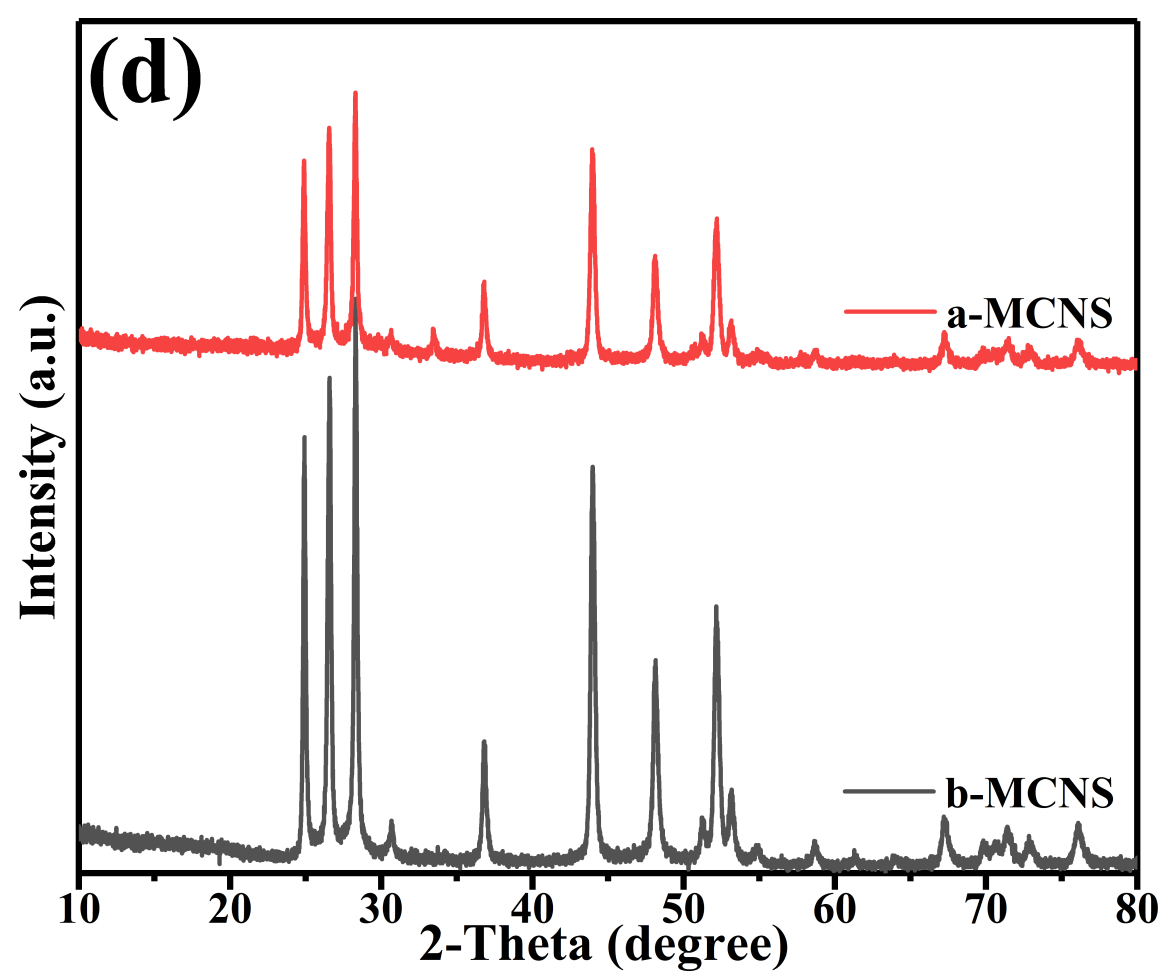

Fig. $6 \mathrm{H}_{2}$ evolution amount of (a) pure $\mathrm{NiSe}_{2}$, MCS with different weight loading ratios of $\mathrm{NiSe}_{2}$; (b) AQE of MCNS-10 in the 400-600 nm wavelength range. (c) The stability test of hydrogen production cycle of MCNS-10; (d) XRD of MCNS-10 composite catalyst after hydrogen production.

\subsection{The PL and TRPL spectra}

In order to study the crucial role of $\mathrm{NiSe}_{2}$ cocatalyst in the boosting the processes of charge separation, transfer and recombination in the composite material MCSN system, the all samples were tested by PL and TRPL spectra.Fig. 7a shown PL spectra of all as-synthesized catalysts with an excitation and emission wavelength of 390 and $533 \mathrm{~nm}$, respectively. The no fluorescence peak was detected in $\mathrm{NiSe}_{2}$, indicating that $\mathrm{NiSe}_{2}$ could not excited by irradiation. The composite catalyst MCNS-10 exhibited the lowest PL intensity compared with other binary composites, indicating that higher separation efficiency of electron-hole pair.

The fluorescence lifetime generally refer to the average time required for an electron to transfer from an excited state to a ground state. The TRPL spectra shown inFig. $\mathbf{7 b}$ further indicated that the charge carriers lifetime of MCNS-10 (2.21 ns) was significantly lower than that of MCS (2.46 ns), MCNS-3 (2.4 ns), MCNS-5 (2.3 ns), MCNS-15 (2.25 ns), MCNS-20 (2.38 ns). Generally, the electron lifetime was negatively correlated with electron transfer rate. The consequences show that the electron transit time of MCNS-10 composite is short [47], this was because $\mathrm{NiSe}_{2}$ could provide additional decay channel to the excited states of the MCNS [43]. We further studied the catalyst performance from two aspects values of electron transfer rate constant $\left(\mathrm{K}_{\mathrm{ET}}\right)$ and the electron injection efficiency $\left(\eta_{\mathrm{inj}}\right)$.

The parameters of emission decay of the samples as the shownTable $\mathbf{2}$. It was clearly observed that $\mathrm{K}_{\mathrm{ET}}$ and $\eta_{\text {inj }}$ value of MCNS-10 were larger than that MCS and other composite catalysts, indicating that $\mathrm{NiSe}_{2}$ acted as electron traps can effectively capture electrons attached on the surface of MCS $[6,45,46]$. Further, three dimensional topography of $\mathrm{NiSe}_{2}$ exposed more active sites and improved the separation efficiency of electrons and hole pairs. These analyses together shown that the $\mathrm{NiSe}_{2}$ play an important role in hydrogen production experiments [25]. 

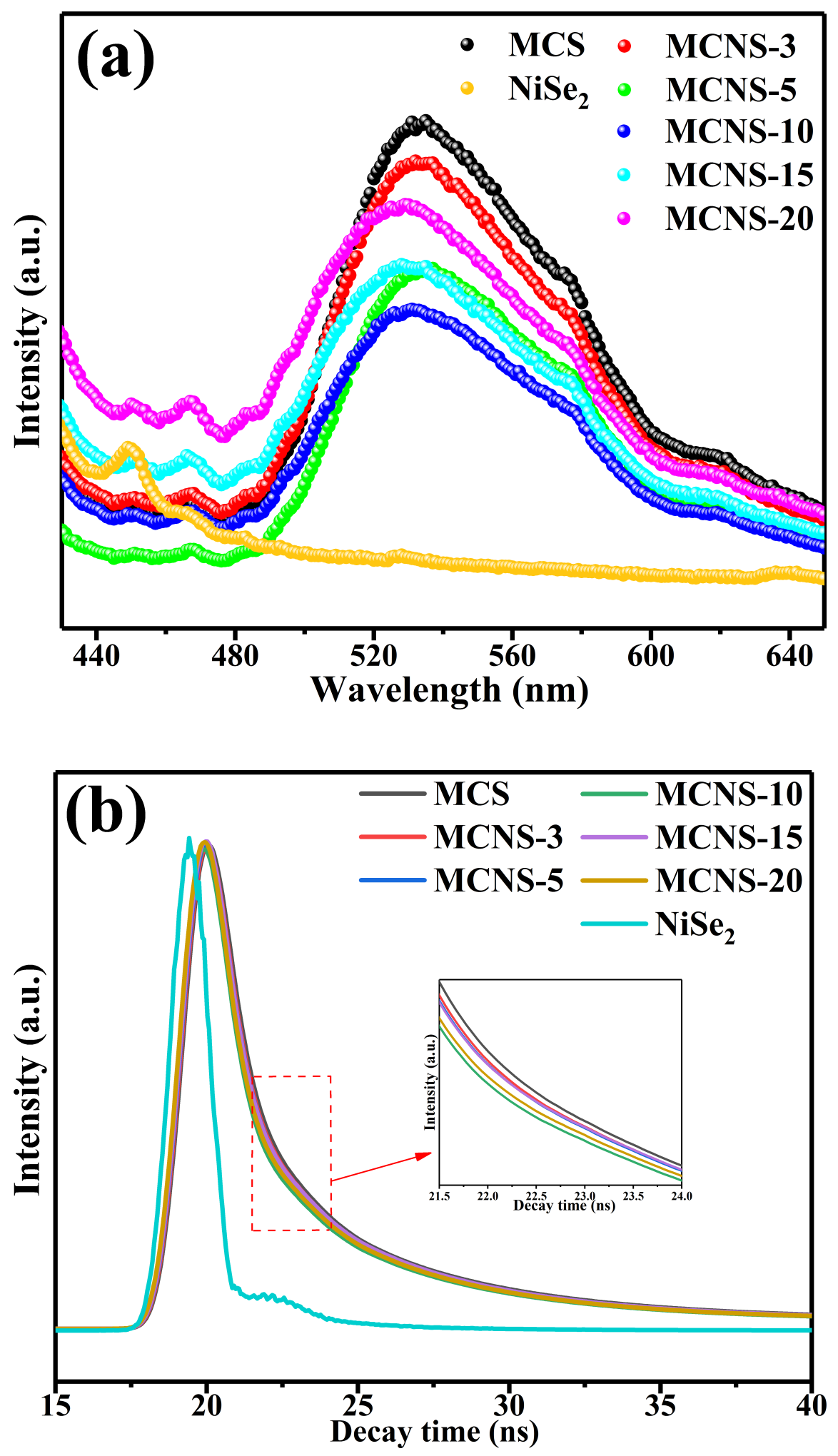

Fig. 7 (a) The PL spectra and (b) the time-resolved photoluminescence spectra of all samples. 
Table 2 The dynamics parameters of electron transfer for samples.

\begin{tabular}{|c|c|c|c|c|c|c|}
\hline Samples & $\tau_{1}[\mathrm{~ns}]$ & $\tau_{2}[\mathrm{~ns}]$ & $\tau_{3}[\mathrm{~ns}]$ & $\tau_{\text {ave }}[\mathrm{ns}]$ & $K_{E T}\left[10^{9} \mathrm{~s}^{-1}\right]$ & $\eta_{(\imath \nu \vartheta)}[\%]$ \\
\hline $\mathrm{MCS}$ & $5.26(30.36 \%)$ & $0.72(24.89 \%)$ & $\begin{array}{l}149.06 \\
(44.75 \%)\end{array}$ & 2.46 & - & - \\
\hline $\mathrm{NiSe}_{2}$ & $0.07(28.65 \%)$ & $0.06(24.39 \%)$ & $4.13(46.96 \%)$ & 0.07 & - & - \\
\hline MCNS-3 & $5.29(29.77 \%)$ & $0.72(25.72 \%)$ & $\begin{array}{l}147.98 \\
(44.51 \%)\end{array}$ & 2.4 & 0.01 & 2.4 \\
\hline MCNS-5 & $5.34(29.51 \%)$ & $0.73(25.68 \%)$ & $\begin{array}{l}149.96 \\
(44.81 \%)\end{array}$ & 2.3 & 0.03 & 6.5 \\
\hline MCNS-10 & $5.25(29.43 \%)$ & $0.67(26.58 \%)$ & $\begin{array}{l}148.03 \\
(43.99 \%)\end{array}$ & 2.21 & 0.05 & 10.2 \\
\hline MCNS-15 & $5.22(29.58 \%)$ & $0.68(26.03 \%)$ & $\begin{array}{l}146.52 \\
(44.38 \%)\end{array}$ & 2.25 & 0.04 & 8.5 \\
\hline MCNS-20 & $5.26(29.68 \%)$ & $0.7(25.31 \%)$ & $\begin{array}{l}148.36 \\
(45.01 \%)\end{array}$ & 2.38 & 0.01 & 3.3 \\
\hline
\end{tabular}

\subsection{Electrochemical analysis}

In this portion, in order to demonstrate the significant function of $\mathrm{NiSe}_{2}$ in facilitating the electron hole separation efficiency, we performed a series of electrochemical characterizations of the MCS, $\mathrm{NiSe}_{2}$ and MCNS-10 samples. Fig. 8a shown the transient photocurrent response spectra of MCS, $\mathrm{NiSe}_{2}$ and MCNS10 in periodic on/off lamp state. Composite catalyst MCNS-10 reveal the highest photocurrent responses, demonstrating that the existence of $\mathrm{NiSe}_{2}$ promoted photogenerated charge separation rate and enhanced visible-light response ability [48]. All samples showed a decreasing trend with the increase of test time, on account of accumulation of holes $[8,24]$.

The LSV was often used to study the carrier characteristics of semiconductor. At higher current densities $(-0.6 \mathrm{~V})$, the LSV curves was shown inFig. 8b . Obviously, $\mathrm{NiSe}_{2}$ acted as co-catalyst can decrease the hydrogen evolution overpotential of MCS . This clearly shown that loading $\mathrm{NiSe}_{2}$ could increase photoinduced electrons participating in hydrogen reaction. Moreover, the Nyquist plots of EIS of MCS, $\mathrm{NiSe}_{2}$ and MCNS10 were shown inFig.8C , the insert was the equivalent circuit diagram, where charge transfer take place in the arched high-frequency region. The diameter of the arc for $\mathrm{NiSe}_{2}$ electrode was the smallest, this was due to the excellent electrical conductivity of $\mathrm{NiSe}_{2}[6,49]$. Secondly, a smaller arc had been detected for composite MCNS-10 compared to that of MCS, indicating a low impedance during charge transfer.

In Fig. 8d, Mott-Schottky plots of MCS and $\mathrm{NiSe}_{2}$ were used to investigate the electrons transport acted of photocatalysis. MCS and MCNS-10 exhibits a positive slopes of the $\mathrm{E}^{-\mathrm{C}^{-2}}$ plots, indicating that they were characteristics n-type semiconductor [16]. The flat-band potential $\left(E_{f b}\right)$ of MCS and $\mathrm{NiSe}_{2}$ could be calculated to be -0.89 and $-0.64 \mathrm{~V}$ versus the saturated calomel electrode (SCE), respectively. According to previous reports, the conduction band $\left(E_{C B}\right)$ values of n-type semiconductor was more negative about 0.1-0.2 V than the $E_{f b}$, and combined with the calculation formula: $E_{N H E}=E_{S C E}+0.24 \mathrm{~V}$ [34]. Therefore, the $E_{C B}$ of MCS and $\mathrm{NiSe}_{2}$ were calculated as -0.85 and $-0.6 \mathrm{eV}$ versus NHE (normal hydrogen electrode). Because $E_{C B}$ value of MCS has a more negative than $\mathrm{NiSe}_{2}$, this indicate that the electron transfer direction was from MCS to $\mathrm{NiSe}_{2}$. 

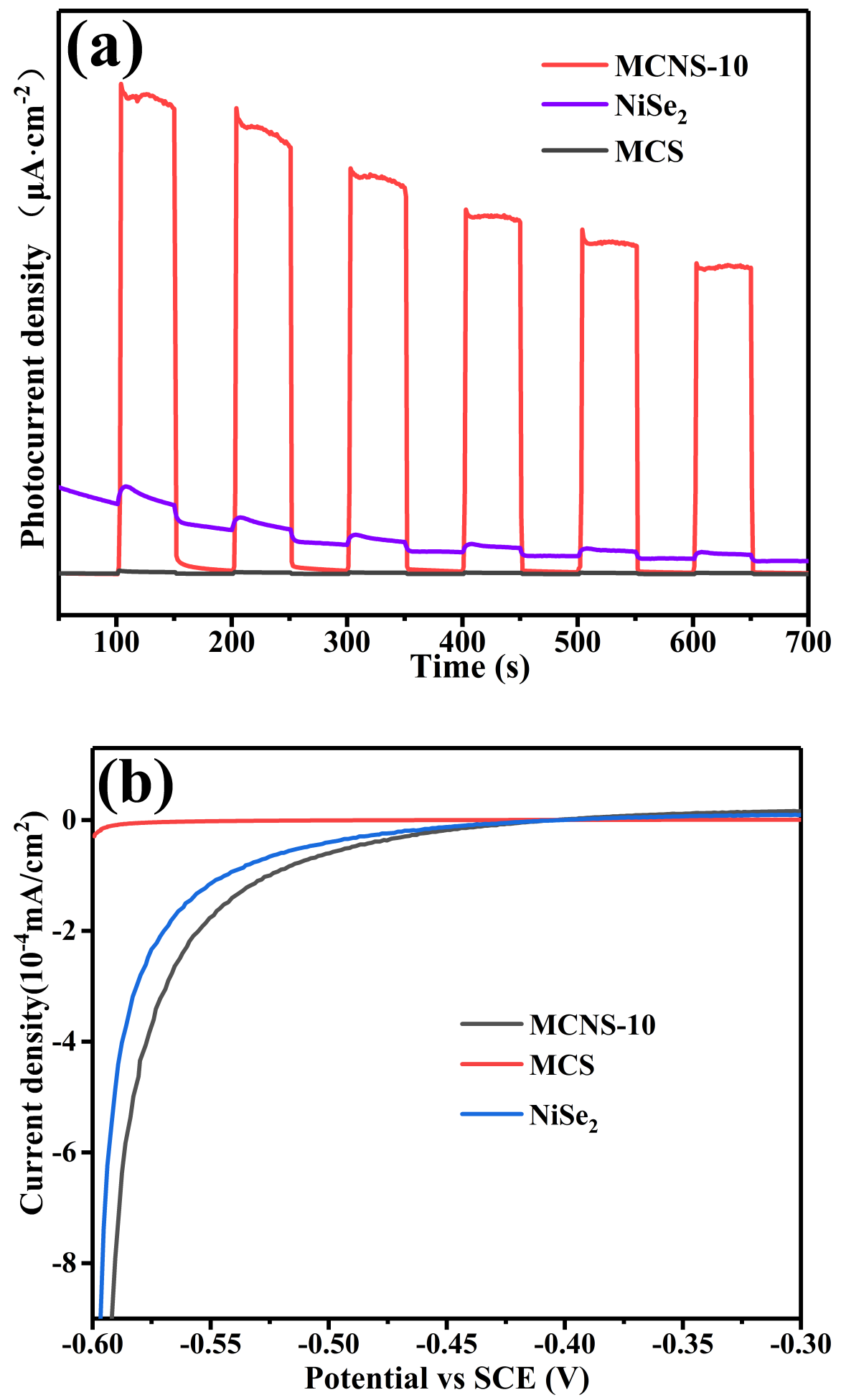

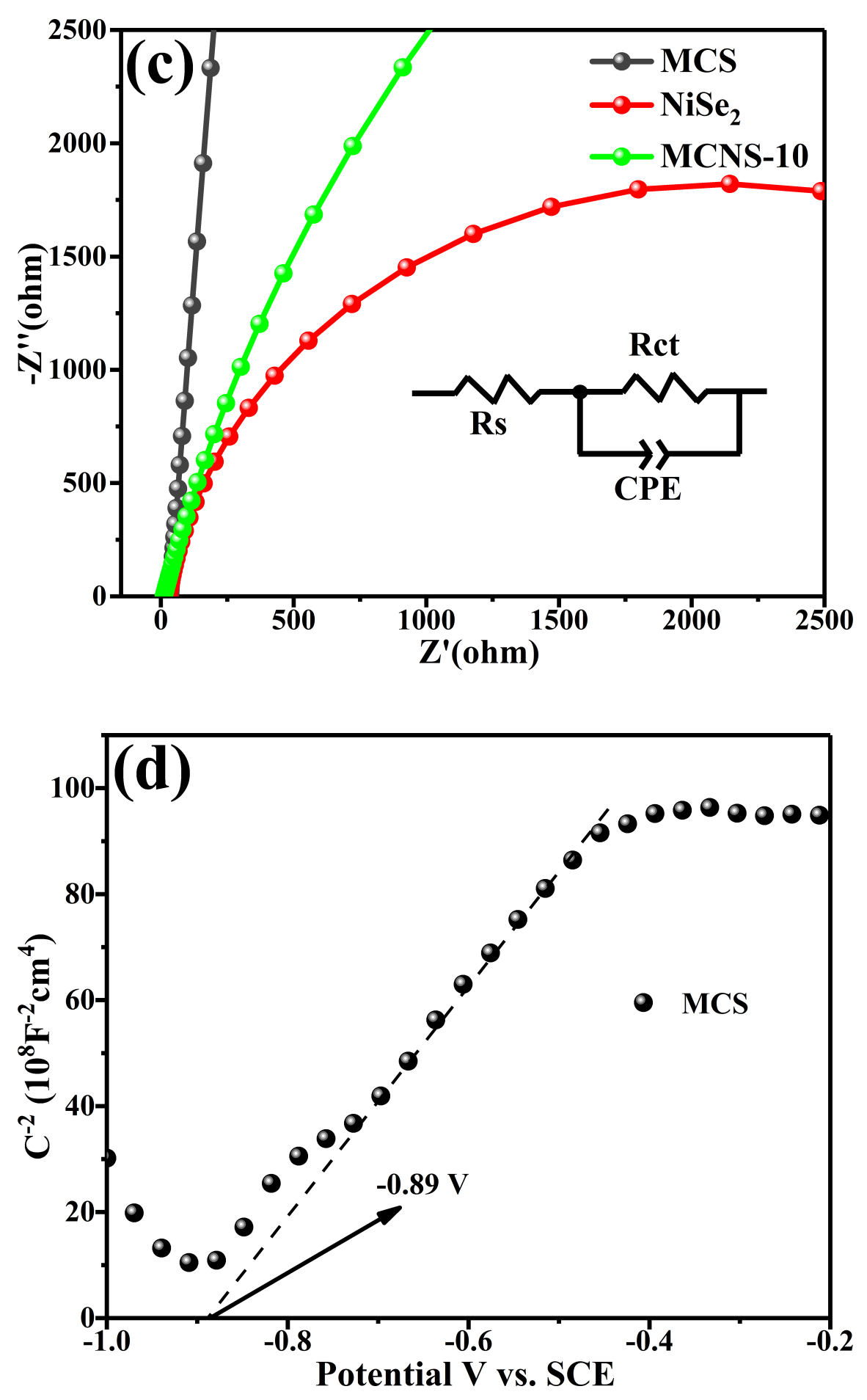


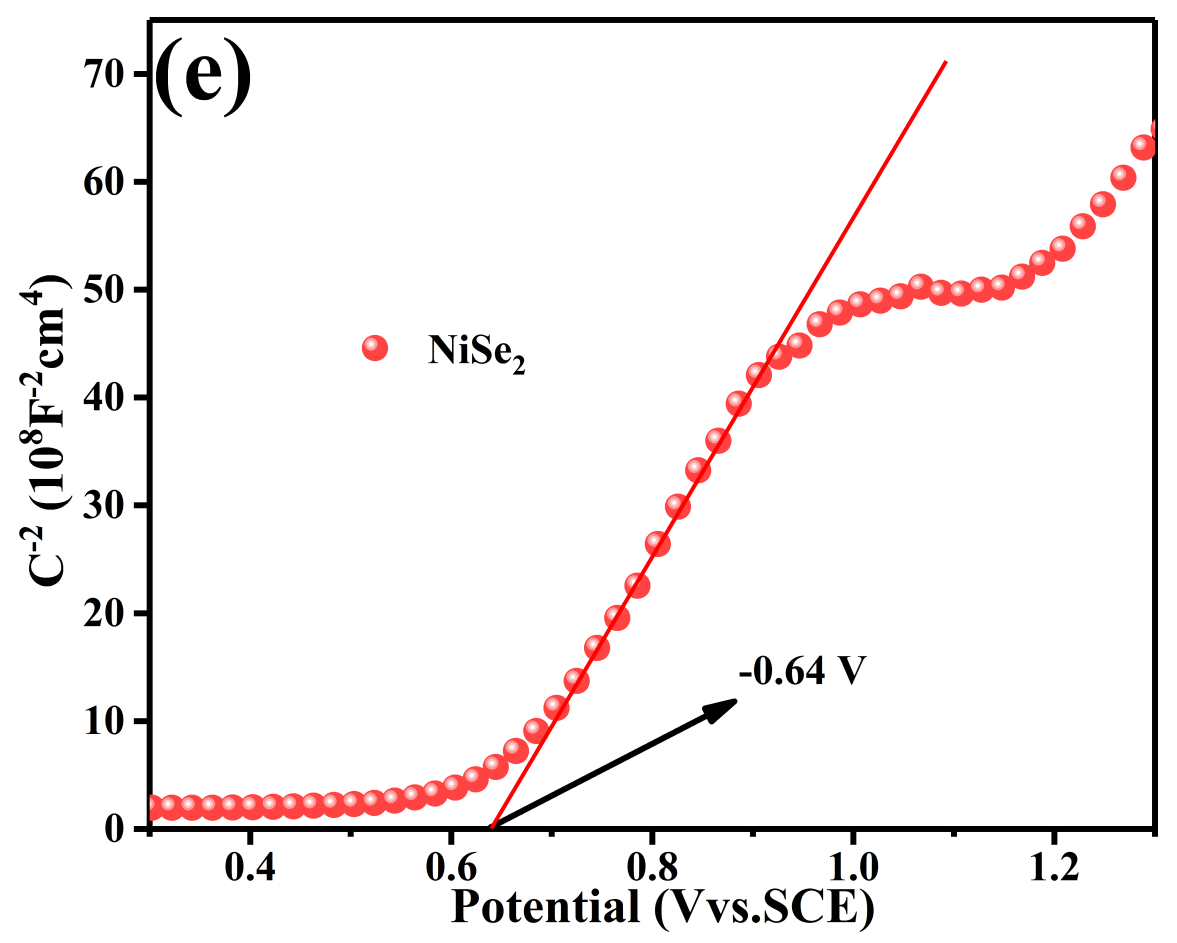

Fig. 8 (a) Transient photocurrent responses, (b)linear scan voltammetry (LSV) curves, (c) electrochemical impedance spectroscopy (EIS) of MCS, NiSe 2 and MCNS-10.(d , e) Mott-Schottky curses of MCS and $\mathrm{NiSe}_{2}$.

\subsection{Proposed reaction mechanism}

Based on all the above results, the charge separation process and proposed mechanism for MCNS-10 was illustrated in Fig. 9 . Under visible light irradiation, the excited state of MCS could be quickly produced electron-hole pairs. The photogenerated electrons of MCS migrate from the VB to $\mathrm{CB}$, and a large number of holes were accumulated on the VB of MCS. The strong reductive $\mathrm{Na}_{2} \mathrm{~S} / \mathrm{Na}_{2} \mathrm{SO}_{3}(0.35 \mathrm{M} / 0.25 \mathrm{M})$ solution was used as sacrificial reagent could consume holes in the VB of MCS, further facilitating charge separation. Since MCS had more negative CB level $(-0.85 \mathrm{eV})$ as compared with the CB potential $(-0.6 \mathrm{eV})$ of $\mathrm{NiSe}_{2}$, the electrons transfer from the $\mathrm{CB}$ of MCS to the $\mathrm{CB}$ of $\mathrm{NiSe}_{2}$ was driven by the thermodynamic driving force [33]. At the same time, 3D cubes $\mathrm{NiSe}_{2}$ can provide more reduction sites, causing $\mathrm{H}^{+}$to combine with electron to generate $\mathrm{H}_{2}$. 


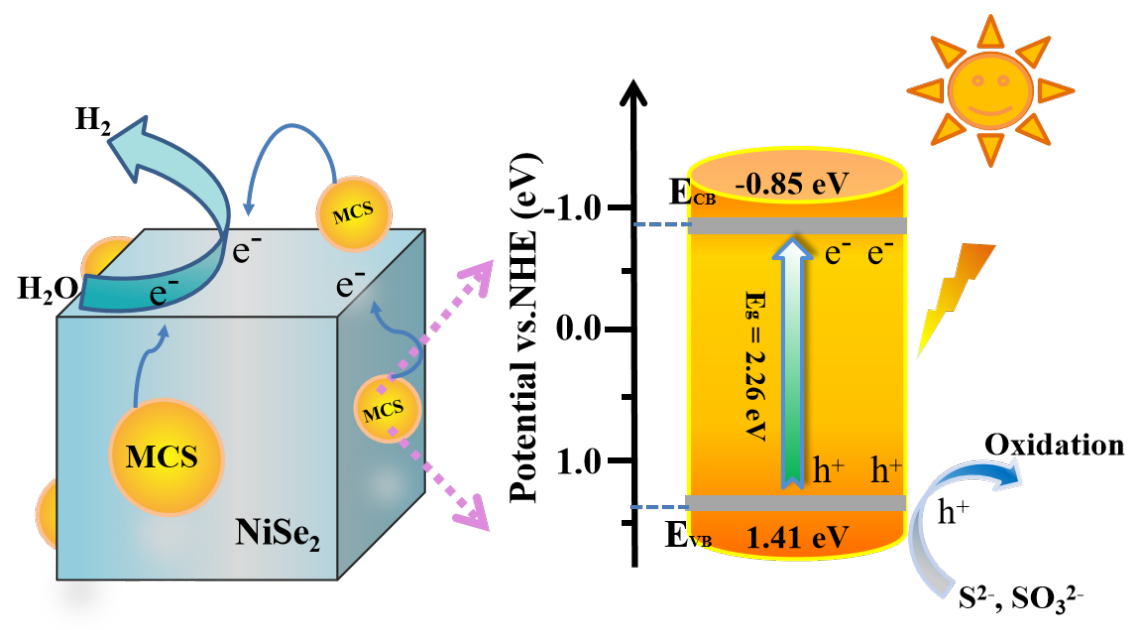

Fig. 9 Schematic illustration of charge transfer for MCNS-10 under visiblelight irradiation.

\section{Conclusion}

In summary, we combined co-catalyst modification and morphological modification to synthesized a new $\mathrm{Mn}_{0.05} \mathrm{Cd}_{0.98} \mathrm{~S} / \mathrm{NiSe}_{2}$ composite catalyst by a facile method. The cubic $\mathrm{NiSe}_{2}$ could provide extensive surface for $\mathrm{Mn}_{0.05} \mathrm{Cd}_{0.95} \mathrm{~S}$ nanoparticles anchoring, effectively preventing the occurrence of $\mathrm{Mn}_{0.05} \mathrm{Cd}_{0.98} \mathrm{~S}$ nanoparticle agglomeration. Secondly, the modification of $\mathrm{NiSe}_{2}$ co-catalyst further improves the light absorption intensity and electrochemical performance of MCS, thereby improving hydrogen production activity. In result, the $\mathrm{Mn}_{0.05} \mathrm{Cd}_{0.98} \mathrm{~S} / \mathrm{NiSe}_{2}$ composite catalyst proved an excellent hydrogen producing activity. The maximum hydrogen production of $713.78 \mu \mathrm{mol}$ is procured over $\mathrm{Mn}_{0.05} \mathrm{Cd}_{0.98} \mathrm{~S} / \mathrm{NiSe}_{2}-10 \mathrm{wt} \%$ composite, which is 7.5 times than that of the pure $\mathrm{Mn}_{0.05} \mathrm{Cd}_{0.95} \mathrm{~S}$. In this work, we designed a series of novel $0 \mathrm{D} / 3 \mathrm{D}$ nanohybrids of $\mathrm{Mn}_{0.05} \mathrm{Cd}_{0.95} \mathrm{~S} / \mathrm{NiSe}_{2}$ photocatalysts, which could beneficial for practical applications.

\section{Author contributions}

Hua Liu and Teng Yan conceived and designed the experiments; Hua Liu performed the experiments; Zhiliang Jin and Qingxiaang Ma contributed reagents/materials and analysis tools; and Hua Liu wrote the paper.

\section{Conflicts of interest}

The authors declare that they have no competing interests.

\section{Acknowledgements}

This work was financially supported by the Open Project of State Key Laboratory of High-efficiency Utilization of Coal and Green Chemical Engineering, Ningxia University (2019-KF-36), the Chinese National Natural Science Foundation $(21862002,41663012)$, the Ningxia low-grade resource high value utilization and environmental chemical integration technology innovation team project, North Minzu University.

\section{Reference:}

1. Xuqiang Hao, Yicong Wang, Jun Zhou, Zhiwei Cui, Ying Wang, Zhigang Zou, Zinc vacancy-promoted photocatalytic activity and photostability of ZnS for efficient visible light driven hydrogen evolution, Applied Catalysis B: Environmental, 2018, 221, 302-311.

2. Jakob Kibsgaard, Thomas F. Jaramillo, Flemming Besenbacher, Building an appropriate active-site motif into a hydrogen-evolution catalyst with thiomolybdate $\left[\mathrm{Mo}_{3} \mathrm{~S}_{13}\right]^{2-}$ clusters, Nature Chemistry, 2014, 6, 248-253. 
3. Jun Zhang, Jiaguo Yu, Yimin Zhang, Qin Li, Jianru Gong, Visible light photocatalytic $\mathrm{H}_{2}$-production activity of $\mathrm{CuS} / \mathrm{ZnS}$ porous nanosheets based on photoinduced interfacial charge transfer, Nano letters, 2011, 11, 4774-4779.

4. Leilei Xu, Haotian Gong, Li Deng, Fei Long, Yu Gu, Jianguo Guan, Complex-mediated synthesis of tantalum oxyfluoride hierarchical nanostructures for highly efficient photocatalytic hydrogen evolution, ACS applied materials and interfaces, 2016, 8, 9395-9404.

5. Bing Luo, Rui Song, Jiafeng Geng, Xiaohe Liu, Dengwei Jing, Menglong Wang, Cheng Cheng, Towards the prominent cocatalytic effect of ultra-small CoP particles anchored on $\mathrm{g}_{-} \mathrm{C}_{3} \mathrm{~N}_{4}$ nanosheets for visible light driven photocatalytic $\mathrm{H}_{2}$ production, Applied Catalysis B: Environmental, 2019, 256, 117819.

6. Hua Liu, Teng Yan, Zhiliang Jin, CoP nanoparticles as cocatalyst modified the CdS/ $\mathrm{NiWO}_{4}$ of p-n heterojunction to produce hydrogen efficiently, New Journal of Chemistry, 2020, 44, 1426-1438.

7. Shaohong Zang, Guigang Zhang, Zhi-An Lan, Dandan Zheng, Xinchen Wang, Enhancement of photocatalytic $\mathrm{H}_{2}$ evolution on pyrene-based polymer promoted by $\mathrm{MoS}_{2}$ and visible light, Applied Catalysis B: Environmental, 2019, 251, 102-111.

8. Sheng Wang, Bicheng Zhu, Mingjin Liu, Liuyang Zhang, Jiaguo Yu, Minghua Zhou, Direct Z-scheme $\mathrm{ZnO} / \mathrm{CdS}$ hierarchical photocatalyst for enhanced photocatalytic $\mathrm{H}_{2}$-production activity, Applied Catalysis B: Environmental, 2019, 243, 19-26.

9. Wenhui Feng, Yaozhu Wang, Xueyan Huang, Kaiqiang Wang, Fan Gao, Yan Zhao, Bo Wang, Lulu Zhang, Ping Liu, One-pot construction of $1 \mathrm{D} / 2 \mathrm{D} \mathrm{Zn}_{1-\mathrm{x}} \mathrm{Cd}_{\mathrm{x}} \mathrm{S} / \mathrm{D}-\mathrm{ZnS}(\mathrm{en})_{0.5}$ composites with perfect heterojunctions and their superior visible-light-driven photocatalytic $\mathrm{H}_{2}$ evolution, Applied Catalysis B: Environmental, 2018, 220, 324-336.

10. Chao Xue, He Li, Hua An, Bolun Yang, Jinjia Wei, Guidong Yang, NiSx quantum dots accelerate electrons transfer in $\mathrm{Cd}_{0.8} \mathrm{Zn}_{0.2} \mathrm{~S}$ photocatalytic system via rGO nanosheet "Bridge" towards superior visible-light-driven hydrogen evolution, ACS Catalysis, 2018, 8, 1532-1545.

11. Shaoqin Peng, Yang Yang, Jiaonv Tan, Chao Gan, Yuexiang Li, In situ loading of $\mathrm{Ni}_{2} \mathrm{P}$ on $\mathrm{Cd}_{0.5} \mathrm{Zn}_{0.5} \mathrm{~S}$ with red phosphorus for enhanced visible light photocatalytic $\mathrm{H}_{2}$ evolution. Applied Surface Science, 2018, 447, 822-828.

12. Xichen Zhou, Zhen Liu, Yifan Wang, Yong Ding, Facet effect of $\mathrm{Co}_{3} \mathrm{O}_{4}$ nanocrystals on visible-light driven water oxidation, Applied Catalysis B: Environmental, 2018, 237, 74-84.

13. Jie Chen, Shaohua Shen, Penghui Guo, Meng Wang, Po Wu, Xixi Wang, Liejin Guo, In-situ reduction synthesis of nano-sized $\mathrm{Cu}_{2} \mathrm{O}$ particles modifying $\mathrm{g}-\mathrm{C}_{3} \mathrm{~N}_{4}$ for enhanced photocatalytic hydrogen production. Applied Catalysis B: Environmental, 2014, 152, 335-341.

14. Qizhao Wang, Yanbiao Shi, Zhongyi Du, Jijuan He, Junbo Zhong, Lianchun Zhao, Houde She, Gang $\mathrm{Liu}$, Bitao Su. Synthesis of rod-like g- $\mathrm{C}_{3} \mathrm{~N}_{4} / \mathrm{ZnS}$ composites with superior photocatalytic activities for degradation methyl orange. European Journal of Inorganic Chemistry, 2015, 24, 4108-4115.

15. Xiaolei Liu, Qi Liu, Peng Wang, Yuzi Liu, Baibiao Huang, Elena A. Rozhkova, Qianqian Zhang, Zeyan Wang, Ying Dai, Jun Lu, Efficient photocatalytic $\mathrm{H}_{2}$ production via rational design of synergistic spatially-separated dual cocatalysts modified $\mathrm{Mn}_{0.5} \mathrm{Cd}_{0.5} \mathrm{~S}$ photocatalyst under visible light irradiation, Chemical Engineering Journal, 2018, 337, 480-487.

16. Qunzeng Huang, Yan Xiong, Qian Zhang, Hongchang Yao, Zhongjun Li, Noble metal-free $\mathrm{MoS}_{2}$ modified $\mathrm{Mn}_{0.25} \mathrm{Cd}_{0.75} \mathrm{~S}$ for highly efficient visible-light driven photocatalytic $\mathrm{H}_{2}$ evolution, Applied Catalysis B: Environmental, 2017, 209, 514-522.

17. Yuanpeng Wang, Guorong Wang, Lijun Zhang, Zhiliang Jin and Tiansheng Zhao, Hydroxides $\mathrm{Ni}(\mathrm{OH})_{2} \& \mathrm{Ce}(\mathrm{OH})_{3}$ as a novel hole storage layer for enhanced photocatalytic hydrogen evolution, Dalton Transactions, 20219, 48, 17660-17672.

18. Hui Zhao, Shengnan Sun, Pingping Jiang, Zhichuan J.Xu, Graphitic $\mathrm{C}_{3} \mathrm{~N}_{4}$ modified by $\mathrm{Ni}_{2} \mathrm{P}$ cocatalyst: An efficient, robust and low cost photocatalyst for visible-light-driven $\mathrm{H}_{2}$ evolution from water, Chemical Engineering Journal, 2017, 315, 296-303.

19. Keita Ikeue, Yumeki Shinmura, Masato Machida, Ag-doped Mn-Cd sulfide as a visible-light-driven photocatalyst for $\mathrm{H}_{2}$ evolution, Applied Catalysis B: Environmental, 2012, 23, 84-88.

20. Qiudi Yue, Yangyang Wan, Zijun Sun, Xiaojun Wu, Yupeng Yuand and Pingwu Du, MoP is a novel, 
noble-metal-free cocatalyst for enhanced photocatalytic hydrogen production from water under visible light, Journal of Materials Chemistry A, 2015, 3, 16941-16947.

21. Hong Liu, Jingchai Meng and Jiang Zhang, Self-assembled three-dimensional flowerlike $\mathrm{Mn}_{0.8} \mathrm{Cd}_{0.2} \mathrm{~S}$ microspheres as efficient visible-light-driven photocatalysts for $\mathrm{H}_{2}$ evolution and $\mathrm{CO}_{2}$ reduction, Catalysis Science Technology, 2017, 7, 3802-3811.

22. Yuexiang Li, Hui Li, Yafei Li, Shaoqin Peng, Yunhang Hu, Fe-B alloy coupled with Fe clusters as an efficient cocatalyst for photocatalytic hydrogen evolution, Chemical Engineering Journal, 2018, 344, 506-513.

23. Zong $\mathrm{Xu}$, Yan Hongjian, Wu Guopeng, Ma Guijun, Wen Fuyu, Wang Lu, Li Can, Enhancement of photocatalytic $\mathrm{H}_{2}$ evolution on CdS by loading $\mathrm{MoS}_{2}$ as Cocatalyst under visible light irradiation, , 2008, 130, 7176-7177.

24. Xinwei Jiang, Haisheng Gong, Qiuwen Liu, Mingxia Song, Caijin Huang, In situ construction of $\mathrm{NiSe} / \mathrm{Mn}_{0.5} \mathrm{Cd}_{0.5} \mathrm{~S}$ composites for enhanced photocatalytic hydrogen production under visible light, Applied Catalysis B: Environmental, 2019, 118439.

25. Guorong Wang, Zhiliang Jin, Function of $\mathrm{NiSe}_{2}$ over CdS nanorods for enhancement of photocatalytic hydrogen production-from preparation to mechanism, Applied Surface Science, 2019, 467, 1239-1248.

26. Xiao Li, Guanqun Han, Yanru Liu, Bin Dong, Wenhui Hu, Xiao Shang, Yongming Chai, Chenguang Liu, NiSe@NiOOH core-shell hyacinth-like nanostructures on nickel foam synthesized by in situ electrochemical oxidation as an efficient electrocatalyst for the oxygen evolution reaction, ACS applied materials \& interfaces, 2016, 8, 20057-20066.

27. Fengmei Wang, Prof. Yuanchang Li, Tofik Ahmed Shifa, Kaili Liu, Feng Wang, Prof. Zhenxing Wang, Peng Xu, Qisheng Wang, Prof. Jun He, Angewandte Chemie International Edition, 2016, 55, 6919-6924.

28. Longzhou Zhang, Yi Jia, Guoping Gao, Xuecheng Yan, Ning Chen, Jun Chen, Mun Teng Soo, Barry Wood, Dongjiang Yang, Aijun Du, Xiangdong Yao,Graphene defects trap atomic Ni species for hydrogen and oxygen evolution reactions, Chem, 2018, 4, 285-297 .

29. Azam Sobhani, Masoud Salavati-Niasari, Fatemeh Davar, Shape control of nickel selenides synthesized by a simple hydrothermal reduction process, Polyhedron, 2012, 31, 210-216.

30. Zhaohui Chen, Haisheng Gong, Qiuwen Liu, Mingxia Song and Caijin Huang, $\mathrm{NiSe}_{2}$ nanoparticles grown in situ on CdS nanorods for enhanced photocatalytic hydrogen evolution, ACS Sustainable Chemistry \& Engineering, 2019, 7, 16720-16728.

31. Na Tiana, Ke Xiao, Yihe Zhang, Xingxu Lu, Liqun Ye, Puxian Gao, Tianyi Ma, Hongwei Huang, Reactive sites rich porous tubular yolk-shell g-C3N4 via precursor recrystallization mediated microstructure engineering for photoreduction, Applied Catalysis B: Environmental, 2019, 253, 196-205.

32. Ting He, Bing Ni, Yangchen Ou, Haifeng Lin, Simin Zhang, Chaozhong Li, Jing Zhuang, Wenping Hu, Xun Wang, Nanosheet-assembled hierarchical carbon nanoframeworks bearing a multiactive center for oxygen reduction reaction, Small Methods, 2018, 2, 1800068.

33. Qunzeng Huang, Zejuan Tao, Liqun Ye, Hongchang Yao, Zhongjun Li, $\mathrm{Mn}_{0.2} \mathrm{Cd}_{0.8} \mathrm{~S}$ nanowires modified by $\mathrm{CoP}_{3}$ nanoparticles for highly efficient photocatalytic $\mathrm{H}_{2}$ evolution under visible light irradiation, Applied Catalysis B: Environmental, 2018, 237, 689-698.

34. Yanbing Li, Zhiliang Jin, Xuqiang Hao, Guorong Wang, Insights into the unique role of cobalt phosphide for boosting hydrogen evolution activity based on MIL-125- $\mathrm{NH}_{2}$, International Journal of Hydrogen Energy, 2019, 44, 17909-17921.

35. Xiaolei Liu, Qi Liu, Peng Wang, Yuzi Liu, Baibiao Huang, Elena A. Rozhkova, Qianqian Zhang, Zeyan Wang, Ying Dai, Jun Lu, Efficient photocatalytic $\mathrm{H}_{2}$ production via rational design of synergistic spatially-separated dual cocatalysts modified $\mathrm{Mn}_{0.5} \mathrm{Cd}_{0.5} \mathrm{~S}$ photocatalyst under visible light irradiation, Chemical Engineering Journal, 2018, 337, 480-487.

36. Jinming Wang Jiang Luo, Dong Liu, Shengtao Chen, Tianyou Peng, One-pot solvothermal synthesis of $\mathrm{MoS}_{2}$-modified $\mathrm{Mn}_{0.2} \mathrm{Cd}_{0.8} \mathrm{~S} / \mathrm{MnS}$ heterojunction photocatalysts for highly efficient visible-light-driven $\mathrm{H}_{2}$ production, Applied Catalysis B: Environmental, 2019, 241, 130-140.

37. Jixiang $\mathrm{Xu}$, Yinhong Qi, Lei Wang, In situ derived $\mathrm{Ni}_{2} \mathrm{P} / \mathrm{Ni}$ encapsulated in carbon $/ \mathrm{g}^{-} \mathrm{C}_{3} \mathrm{~N}_{4}$ hybrids 
from metal-organic frameworks/g- $\mathrm{C}_{3} \mathrm{~N}_{4}$ for efcient photocatalytic hydrogen evolution, Applied Catalysis B: Environmental, 2019, 246, 72-81.

38. Yuanzi Xu, Chengzong Yuan, Xueping Chen, Co-Doped NiSe nanowires on nickel foam via a cation exchange approach as efficient electrocatalyst for enhanced oxygen evolution reaction, RSC advances, 2016, 6, 106832-106836.

39. Tongtong Wang, Daqiang Gao, Wen Xiao, Pinxian Xi, Desheng Xue \& John Wang, Transition-metaldoped $\mathrm{NiSe}_{2}$ nanosheets towards efficient hydrogen evolution reactions, Nano Research, 2018, 11, 60516061.

40. Yang Yang, Yikun Kang, Huihui Zhao, Xiaoping Dai, Meilin Cui, Xuebin Luan, Xin Zhang, Fei Nie, Ziteng Ren, and Weiyu Song, An interfacial electron transfer on tetrahedral $\mathrm{NiS}_{2} / \mathrm{NiSe}_{2}$ heterocages with dual-phase synergy for efficiently triggering the oxygen evolution reaction, Small, 2020, 16, 1905083.

41. Yue Cao, Guorong Wang, Qingxiang Ma and Zhiliang Jin, An amorphous nickel boride-modified ZnxCd1-xS solid solution for enhanced photocatalytic hydrogen evolution, Dalton Transactions, 2020, 49, 1220.

42. Xian Yan, Zhiliang Jin, Yupeng Zhang, Yongke Zhangaand and Hong Yuan, Sustainable and efficient hydrogen evolution over a noble metal-free WP double modified $\mathrm{ZnxCd}_{1-\mathrm{x}} \mathrm{S}$ photocatalyst driven by visible-light, Dalton Transactions. 2019, 48, 11122-11135.

43. Xiangbin Meng, Jingli Sheng, Hongliang Tang, Xiaojun Sun, Hong Dong, Fengming Zhang, MetalOrganic framework as nanoreactors to Co-incorporate carbon nanodots and CdS quantum dots into the pores for improved $\mathrm{H}_{2}$ evolution without noble-metal cocatalyst, Applied Catalysis B: Environmental, 2019, 244, 340-346.

44. Chenyang Zhang, Huanhuan Liu, Wanni Wang, Haisheng Qiana, Sheng Cheng, Yang Wang, Zhengbao Zha, Yijun Zhong, Yong $\mathrm{Hu}$, Scalable fabrication of $\mathrm{Zn}_{\mathrm{x}} \mathrm{Cd}_{1-\mathrm{x}} \mathrm{S}$ double-shell hollow nanospheres for highly efficient hydrogen production, Applied Catalysis B: Environmental, 2018, 239, 309-316.

45. Zizheng Ai, Yongliang Shao, Bin Chang, Lei Zhang, Jianxing Shen, Yongzhong Wu, Baibiao Huang, Xiaopeng Hao, Rational modulation of p-n homojunction in P-doped g- $\mathrm{C}_{3} \mathrm{~N}_{4}$ decorated with $\mathrm{Ti}_{3} \mathrm{C}_{2}$ for photocatalytic overall water splitting, Applied Catalysis B: Environmental, 2019, 259, 118077.

46. Yumin Liu, Hao Ren, Hua Lv, Zhiyuan Gong, Yafei Cao, An insight into the trifunctional roles of $\mathrm{Cu}_{2}(\mathrm{OH})_{2} \mathrm{CO}_{3}$ cocatalyst in boosting the photocatalytic $\mathrm{H}_{2}$ evolution activity over $\mathrm{Zn}_{0.5} \mathrm{Cd}_{0.5} \mathrm{~S}$ nanoparticles, Applied Surface Science, 2019, 484, 1061-1069.

47. Qunzeng Huang, Zejuan Tao, Liqun Ye, Hongchang Yao, Zhongjun Li, $\mathrm{Mn}_{0.2} \mathrm{Cd}_{0.8} \mathrm{~S}$ nanowires modified by $\mathrm{CoP}_{3}$ nanoparticles for highly efficient photocatalytic $\mathrm{H}_{2}$ evolution under visible light irradiation, Applied Catalysis B: Environmental, 2018, 237, 689-698.

48. Sibo Wang, Yan Wang, Song Lin Zhang, Shuang-Quan Zang, Xiong Wen (David) Lou, Supporting ultrathin $\mathrm{ZnIn}_{2} \mathrm{~S}_{4}$ nanosheets on $\mathrm{Co} / \mathrm{N}$-Doped graphitic carbon nanocages for effcient photocatalytic $\mathrm{H}_{2}$ generation, 2019, 31, 1903404.

49. Qiaohong Zhu, Bocheng Qiu, Huan Duan, Yeteng Gong, Zengwei Qin, Bin Shen, Mingyang Xing, Jinlong Zhang, Electron directed migration cooperated with thermodynamic regulation over bimetallic $\mathrm{NiFeP} / \mathrm{g}-\mathrm{C}_{3} \mathrm{~N}_{4}$ for enhanced photocatalytic hydrogen evolution, Applied Catalysis B: Environmental, 2019, 259, 118078 . 